\title{
Erasmus Plus. Se rencontrer pour s'enrichir
}

\section{Elena Maria Grosso}

\section{OpenEdition}

\section{Journals}

Édition électronique

URL : http://journals.openedition.org/esp/3023

DOI : 10.4000/esp.3023

ISSN : 2532-0319

\section{Éditeur}

Centre d'Information sur l'Éducation Bilingue et Plurilingue

\section{Édition imprimée}

Date de publication : 1 décembre 2018

Pagination : 33-41

ISSN : 1127-266X

\section{Référence électronique}

Elena Maria Grosso, «Erasmus Plus. Se rencontrer pour s'enrichir », Éducation et sociétés plurilingues [En ligne], 45 | 2018, mis en ligne le 01 décembre 2020, consulté le 04 décembre 2020. URL : http:// journals.openedition.org/esp/3023 ; DOI : https://doi.org/10.4000/esp.3023 


\section{ERASMUS PLUS. SE RENGONTRER POUR S'ENRIGHIR}

\section{Elena Maria Grosso et alii.}

L'Unione europea, grazie ai suoi progetti, offre sempre possibilità maggiori di scambio e di confronto. Anche alcune scuole della Valle d'Aosta hanno colto questa apertura offrendo ad alunni e insegnanti momenti di formazione e di nuove conoscenze a contatto con altre realtà. Da qui un arricchimento non solo in diversi ambiti disciplinari ma un evidente rinforzo di competenze trasversali tradotte in visione sistemica, problem solving, comunicazione, collaborazione, motivazione, pianificazione e innovazione.

Parole chiave: Plurilinguismo, educazione sostenibile, nuove tecnologie

The projects of the European Union offer ever greater possibilities for exchanges and comparisons. Some schools in the Aosta Valley have seized these opportunities by offering pupils and teachers training to enhance their knowledge through contact with other realities. Hence a personal and professional enrichment not only in various disciplinary fields but a clear reinforcement of soft skills that translate into a systemic vision, problem solving, communication, collaboration, motivation, planning and innovation.

Key-words: Multilingualism, sustainable education, new technologies

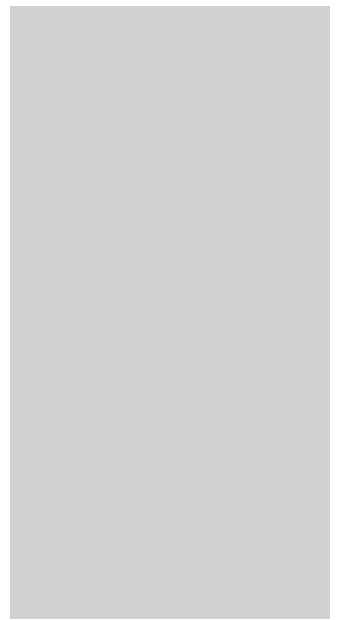

«Plurilinguisme, développement durable et numérique» réunissent quatre régions européennes dans lesquelles six langues sont parlées. Erasmus Plus, Parcours TICE, L'éducation au développement durable dans nos langues est un programme qui permet à des enseignants et des élèves des régions Occitanie (France), Val d'Aran (Espagne), Vallé d'Aoste et Piémont (Italie) de se rencontrer et de collaborer sur l'éducation au développement durable et sur l'utilisation des nouvelles technologies. Tous les participants enseignent et apprennent dans un environnement scolaire bi/plurilingue, où les cours sont dispensés dans deux langues ou plus.

Financé dans le cadre de l'action Partenariats stratégiques pour l'enseignement scolaire du programme européen Erasmus +, le projet de deux ans prévoyait trois séminaires transfrontaliers de formation d'enseignants, trois échanges scolaires entre élèves de 11-13 ans, et des projets collaboratifs menés dans les classes pour l'élaboration de jeux numériques.

Lancé à l'automne 2016 à Aoste, la réflexion de la première ren- 


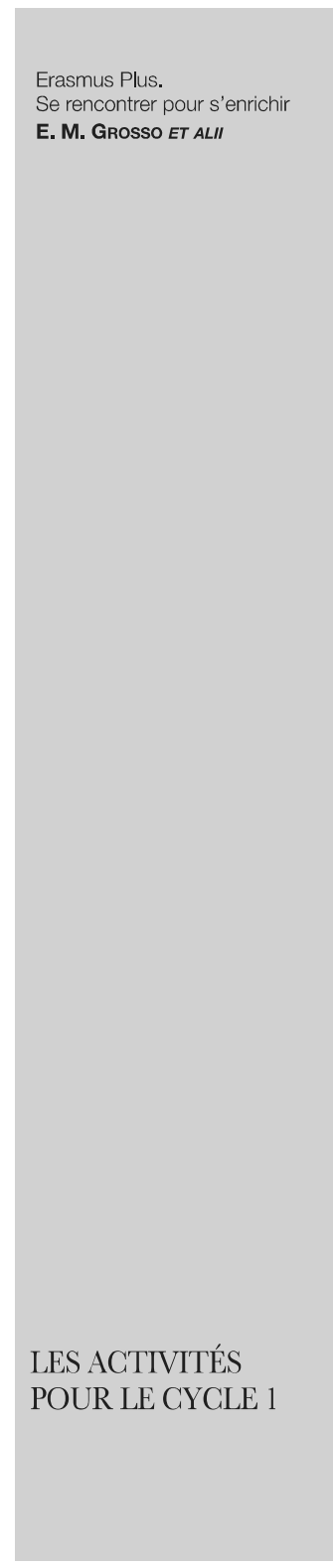

contre a été dédiée à l'interconnaissance, à un approfondissement des différents systèmes scolaires et de la situation linguistique de chaque pays, aux enjeux didactiques du plurilinguisme, aux rapports homme-montagne. Elle s'est poursuivie à Carcassonne au mois de mars 2017 et au Val d'Aran au mois de novembre 2017. Ici, le partage d'expériences didactiques, des conférences sur l'environnement et des ateliers pratiques sur le plurilinguisme et l'enseignement, des observations sur l'apport de l'intercompréhension au niveau des acquisitions disciplinaires et sur l'intégration de l'intercompréhension dans les jeux numériques (voir à la fin de l'article EDD et intercompréhension), ont permis aux participants de micux tracer la route du projet et de construire des outils de travail. Et les deux rencontres - en mai et en novembre 2017 - en Vallée d'Aoste et au Val d'Aran, ont permis aux jeunes élèves espagnols, italiens et français d'utiliser toutes les langues, français, espagnol, italien, occitan, francoprovençal, aranais dans un vrai contexte communicatif, en facilitant la connaissance réciproque et l'ouverture aux autres.

Du point de vue pédagogique, enseignants et conseillères pédagogiques ont constitué trois groupes de travail - un pour chaque cycle scolaire - qui, selon l'âge des enfants, ont mené une réflexion sur les activités à proposer dans les classes, et sur comment évaluer les connaissances et compétences initiales et finales. Des tests sur l'EDD (Éducation au développement durable), l'intercompréhension des langues et les TICE (Technologies de l'Information et de la Communication pour l'Enseignement) ont été élaborés et proposés régulièrement aux élèves, afin de vérifier l'efficacité des interventions et l'avancement du projet. L'éducation durable, les langues et l'emploi du numérique étant les fils conducteurs du projet, les participants ont convenu de créer des comptines et des récits, où les enfants - quel que soit leur âge - par le biais d'animaux cheminant à travers l'Italie, la France et l'Espagne, découvrent, par des jeux numériques, des langues différentes et, par-là, le territoire, ses ressources et la façon de les préserver. Learning Apps et Scratch ont été les programmes le plus souvent utilisés.

Pour les enfants de l'école maternelle de 5-6 ans, la première phase du projet a proposé des jeux de plateau et de simples jeux numériques. Des noms concernant des domaines que les petits connaissent bien - environnement proche de l'école, objets de la cour, produits du jardin - ont été présentés en différentes langues et associés à des images de découverte. L'oralité a été privilégiée; 


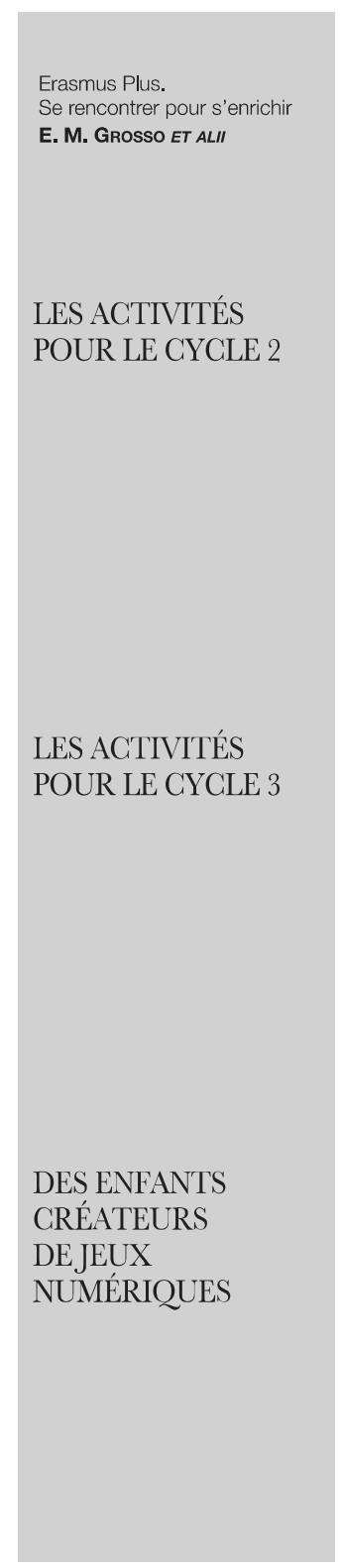

les consignes ont été formulées dans la ou les langues d'enseignement. Des enregistrements sonores effectués par les partenaires du projet ont facilité l'appréhension des différences et des similitudes entre les langues: langues que les enfants ont dû essayer de reconnaitre et de reproduire, langues qui, même si pas toujours familières, ont permis de comprendre de simples énoncés en partant d'un contexte donné.

Nourriture et éléments naturels ont été le fil conducteur des jeux proposés aux enfants du deuxième cycle de 7-9 ans: ce sont les aventures d'une abeille qui leur permettent d'entrer en contact avec l'eau, le vent et la lumière, sources de vie et de bonheur. Jeux de paires, de tri, textes à trou et puzzle de classement ont fait se rencontrer occitan aranais, occitan languedocien, français, espagnol et italien, leurs sonorités et leurs formes écrites. Exercices de repérage, de comparaison, de communication orale ont renforcé les acquis en permettant aux élèves de jouer avec des mots étrangers et inconnus, de comprendre la même histoire racontée en différentes langues.

Enfin, pour les élèves un peu plus âgés, de 10 à 13 ans, les jeux construits ont permis la découverte des pays participant au projet, de leur culture et des sources d'énergie renouvelable. En visitant divers milieux, Petit Ours, le protagoniste de ces jeux réalisés par les enseignants sous Learning Apps, en apprécie les ressources naturelles qui doivent être respectées pour préserver l'environnement. Ici, les jeux n'ont pas seulement ciblé l'intercompréhension mais surtout les savoirs disciplinaires de l'EDD, renforcés par les expériences authentiques vécues lors des échanges des classes qui ont permis aux collégiens du Val d'Aoste, du Val d'Aran et de Marvejols (Occitanie) de se rencontrer tout au long du projet.

Si la première année du projet a vu enseignants et formateurs s'essayer à la construction des jeux numériques et réfléchir sur les modalités d'évaluation des compétences acquises ou à acquérir par les élèves, la deuxième année a permis aux élèves de devenir créateurs eux-mêmes de jeux numériques et d'utiliser les notions apprises pendant la première phase du projet.

«De la table à la poubelle, de la poubelle au jardin, du jardin à la table»: en suivant cette progression, les plus petits ont réalisé des jeux de plateau sur le jardin au fil des saisons. $\grave{A}$ l'aide des adultes, ces derniers ont été transformés en jeux numériques accompagnés de photos choisies ou faites par les enfants, et de leurs voix enre- 


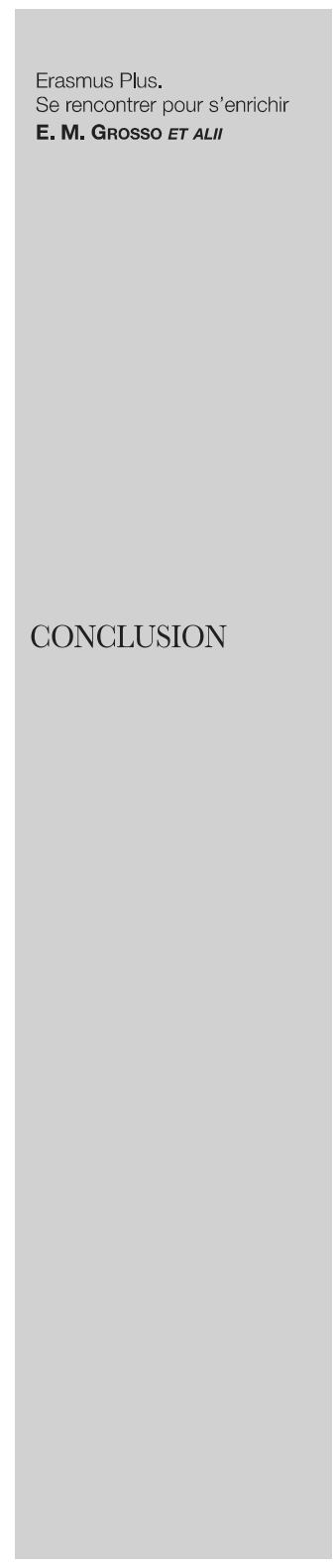

gistrées. Les élèves du cycle 2 ont approfondi les sujets concernant la santé dans l'assiette et les propriétés des aliments, tandis que ceux du cycle 3 ont réfléchi sur le comportement éco-responsable - recyclage, traitements des déchets et des eaux. Des ateliers, où les élèves collaborent en partageant leurs expériences, ont permis la création de nouveaux jeux numériques accompagnés de fiches descriptives et l'acquisition de nouvelles compétences. Les connaissances ont été en outre renforcées par l'élaboration de fiches techniques d'approfondissement sur l'environnement et ses ressources exploitables.

Et à ne pas oublier l'atout du projet: le travail de groupe et en présence - grâce aux échanges scolaires - et en ligne, par le biais d'espaces de travail partagés. Ce qui a permis de se confronter et de tracer, chaque fois, une nouvelle route en l'adaptant à la spécificité des élèves, à leurs intérêts, à leur savoir-être et à leurs savoir-faire.

Le projet se solde par un bilan positif sur toute la ligne. En plus des compétences que les élèves et les enseignants ont acquises, des contacts et des liens qui se sont créés - et qui seront peut-être la raison de poursuivre la collaboration dans d'autres domaine et d'autres initiatives - le projet Erasmus Plus «L'EDD dans nos langues» a permis la réalisation d'un nombre important d'outils en plusieurs langues: jeux, activités clés en main, fiches pédagogiques... Tous ces outils seront diffusés dans les classes et progressivement disponibles sur le portail collectif Occitanica, administré par le CIRDOG (www.occitanica.eu/maleta.occitanica.eu), et mis à la disposition d'autres enseignants, d'autres équipes pédagogiques.

Cela assurera la dissémination et la pérennisation du projet qui pourra être aussi expérimenté dans d'autres classes et dans d'autres régions intéressées.

\section{Erasmus Plus. Témoignages (coordination Elena Maria Grosso)}

Les témoignages et les activités de classe qui suivent racontent comment les élèves ont mis en pratique certaines compétences et comment le projet a visé l'acquisition d'autonomie, de responsabilité, de réflexion, d'esprit pratique, de sens civique, de socialisation et de collaboration. Les activités, proposées d'abord en première langue de scolarisation, s'ouvrent au plurilinguisme par des exercices d'enrichissement lexical, des récits de l'expérience vécue, par le partage du travail réalisé avec les élèves des autres pays participant au projet. 


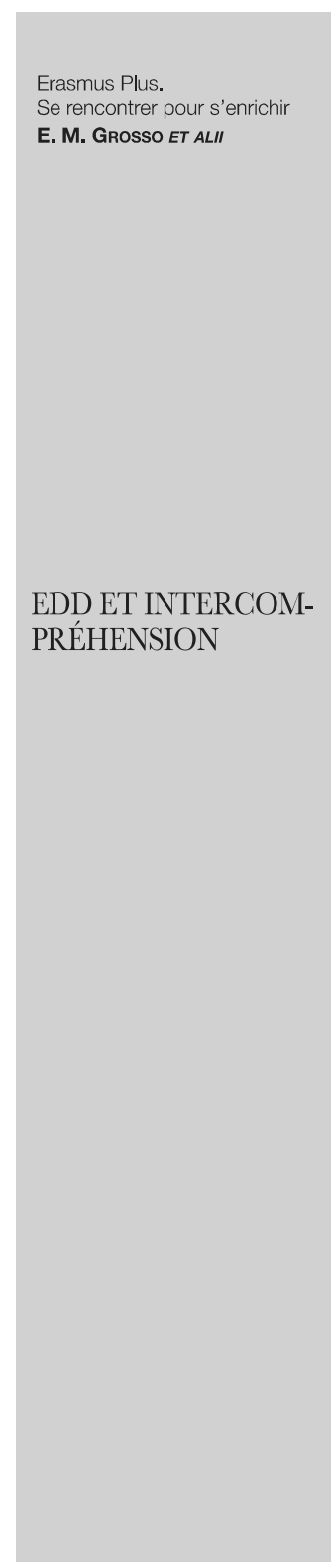

Toutes les langues du projet sont mises en contribution pour favoriser l'ouverture aux langues et les stratégies d'intercompréhension entre langues ayant la même origine. C'est la raison pour laquelle, dans les textes qui suivent, nous avons cherché à proposer un échantillon de cette diversité linguistique, en utilisant les langues du projet, accompagnées d'une brève description en français; nous invitons les lecteurs à se mettre en jeu, comme l'ont fait nos élèves tout au long du projet, pour essayer de comprendre.

Le premier témoignage est une activité de classe en aranais présentée aux élèves dans les autres langues du projet; le deuxième fait le point sur la valeur du travail de groupe et sur l'emploi de plusieurs langues dans différents contextes communicatifs; le dernier présente une activité où des élèves de l'école secondaire deviennent tuteurs des plus petits pour leur faciliter l'élaboration de jeux numériques en plusieurs langues.

\section{Carmina POMAR}

En partant de la visite de la station d'épuration de Vielha et de Mijaran on a proposé aux élèves de la classe de $5^{\mathrm{e}}$ du niveau primaire (10 ans), l'Escola Garona de Vielha (Val d'Aran), des activités pour les faire réfléchir sur l'utilité de l'eau, ce qu'ils ont fait dans leur langue (aranais):

adaiguar per gotejament, adaiguar jardins e plantes, hèr a béuer es animaus, adaiguar es arbes frutèrs e ortalisses, heiredar es maquines, lauar era vaishèra,tintar era ròba,l auar es pàrrecs des animaus, codinar, hèr activitals esportiues, nelejar es animaus, produsir electricilal emplegar tecniques d'inondacion enes camps d'arròs.

Puis on a approfondi les thématiques de l'emploi de l'eau et de l'épuration des eaux non potables.

La planification des activités a suivi le schéma suivant:
1. utilitats dera aigua
2. estauviam era aigua
3. era aigua, abantes e dempús d'èster utilizada
4. eth cicle dera aigua (le cycle de l'eau)
5. era depuracion des aiguies
6. investigam: era nòsta depuradora
7. sabies que...? qué è aprenut?
8. materiau audiovisuau

L'étude du territoire a permis, en même temps, l'acquisition de sujets scientifiques (cycle de l'eau) et proposé une expérimentation pratique pour remettre en jeu les contenus appris.

Ici, les consignes en aranais pour construire un dépurateur d'eau 


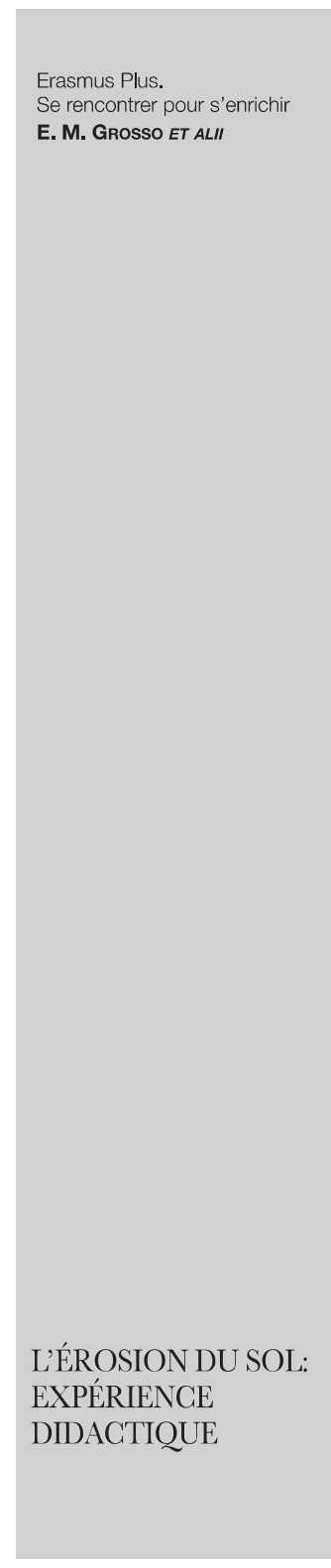

fait à la maison; les élèves pourront écouter les mêmes consignes en italien, occitan et français.

Fiche élève (en aranais)

\section{CONSTRUCCION D'UA DEPURADORA CASALÈRA}

Materiau: Ampolha d'aigua talhada, Pèires de diuèrsa mesura, Grava, Sable, Huelhes, Aigua lorda, Coton

\section{Procediment:}

1. a. Plaçam era ampolha bocaenjós talhada peth miei

2. b. Començam a bastir es coches dera nòsta depuradora:

-Celh de coton

-Gelh de pèires de mès granes a mès petites.

-Celh de sable

-Huelhes.

3. c. Vessam era aigua lorda ena nòsta depuradora e veiram coma se filtre e ges aigua depurada e neta.

\section{Investigam:}

Un viatge as acabat era tua practica, penses qu'ei çò de madeish aigua depurada qu'aigua potabla?

\section{Sabies que:}

Ua gota d'aigua tarde 4 dies, naut o baish, des de qu'entre ena planta depuradora enquia que'n ges.

Existís tecnologia entà transformar era aigua residuau en aigua potabla.

Era aigua qu'entre ena planta ei cargada de bacteries que posteriorament intervieràn ena part biologica deth procès de depuracion.

Era flaira que despren era aigua lorda proven des gasi qu'emelen es bacteries presentes ena aigua.

Es tovalholetes igieniques provòquen grèus problèmes enes filtres des depuradores, pr'amor que non se des hèn ena aigua e caperen es horats des sedac

\section{Que as aprenut...?}

C'est l'expérience pratique qui fait le mieux apprendre aux élèves travaillant ensemble en utilisant, par jeu, des mots et expressions d'autres langues.

\section{Paola FIORI, Anna MarTin}

La classe de 2 ème C de l'Institution scolaire "Maria Ida Viglino", de Villeneuve, Val d'Aoste, a pu approfondir des aspects concernant l'environnement et comparer différentes expériences: la mesure du $\mathrm{pH}$ du sol et de la pluie, la tension de surface, la per- 


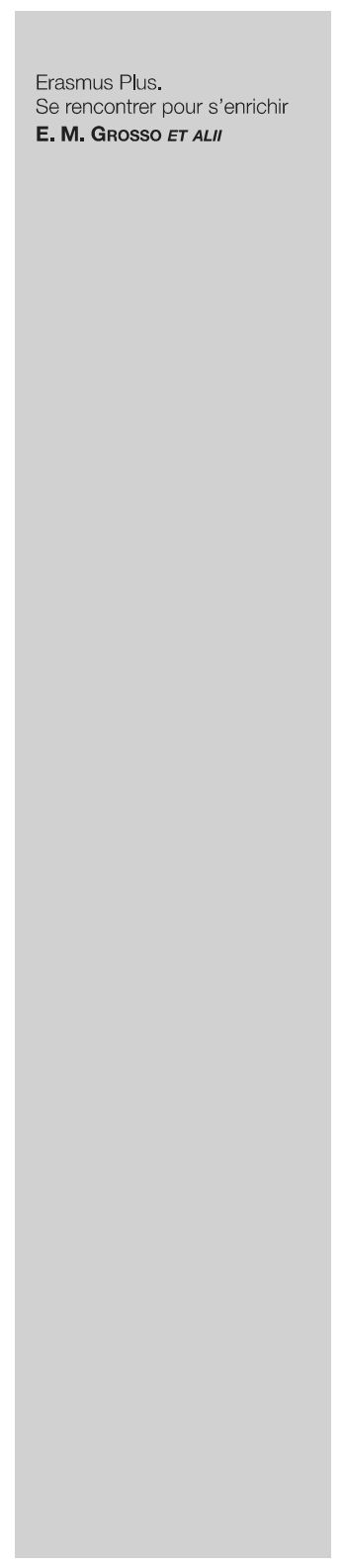

méabilité et l'érosion du sol. Les élèves ont été divisés en quatre groupes, un pour chaque expérience à réaliser. Ils ont réfléchi sur les objectifs de la tâche qui leur avait été confiée et ils se sont organisés pour récupérer le matériel utile à sa réalisation. Ensuite, ils ont effectué l'expérience en s'entraînant à expliquer à haute voix la procédure. Ils ont conçu deux exercices de compréhension: dans le premier, ils ont inséré une liste où il fallait récupérer les outils employés, dans le deuxième, ils ont produit un texte à trous sur le mode opératoire. Ensuite, ils ont réalisé des fiches à l'aide de photos qui expliquaient la démarche dans sa globalité.

L'expérience et l'évaluation ont été soumises aux trois autres groupes et, à travers une réflexion commune, quelques petits ajustements ont été apportés.

Enfin, lors de la visite organisée dans le cadre du projet Erasmus, les élèves espagnols, divisés en petits groupes, ont pu participer à chaque expérience, suivre l'explication de leurs correspondants italiens et vérifier leur compréhension à travers les exercices préparés. Un bilan final, qui a relevé les points forts et faibles, a été fait en classe le lendemain.

$\mathrm{Au}$ cours d'une phase successive, des élèves de l'école secondaire sont devenus tuteurs des plus petits pour les faciliter dans l'élaboration de jeux numériques en plusieurs langues: de nouvelles compétences sont mises en jeu.

\section{Fiche didactique}

\section{L'érosion du sol}

Contexte: l'eau est un agent d'érosion. Les risques augmentent si le sol est composé de résidus. La végétation protège le sol de l'impact de la pluie, ralentit la vitesse de l'eau de ruissellement et permet une meilleure infiltration. Le ruissellement se produit quand l'eau sur une pente ne peut pas s'infiltrer assez vite dans le sol ou être interceptée par des obstacles naturels. Certaines conditions apportées par le changement climatique, telles que les fortes précipitations, accroissent les risques d'érosion.

Objectifs: reconnaître les différents types d'érosion, découvrir les liens entre les impacts du changement climatique et l'érosion.

Matériel: trois bouteilles en plastique, des cailloux, un arrosoir, de l'eau, des résidus de plantes mortes (brindilles, feuilles sèches d'écorce, racines), un terrain avec des plantes, de la terre nue et sèche. 


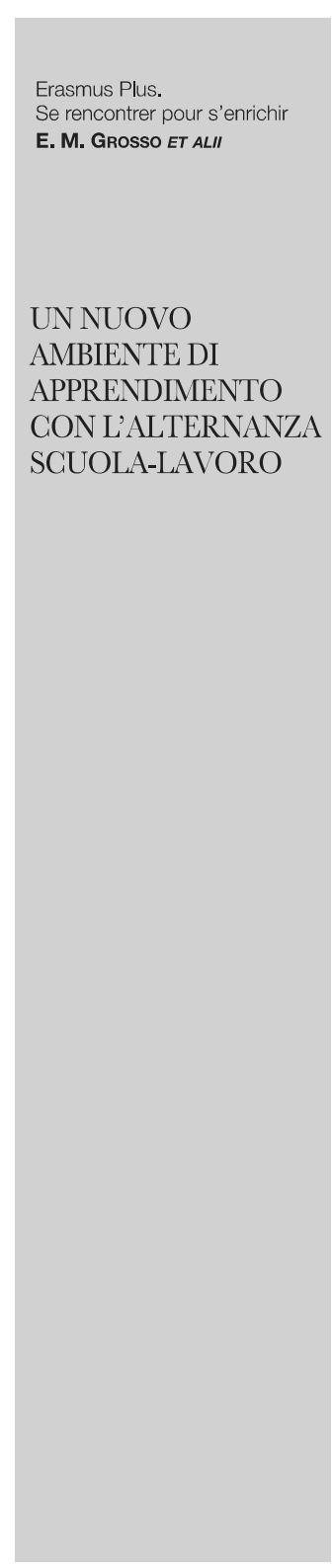

Procédure: les trois bouteilles ont été remplies différemment, l'une avec du terrain et de l'herbe, l'autre avec des résidus et la dernière avec de la terre nue. On a versé la même quantité d'eau dans chaque bouteille et on a attendu qu'elle s'écoule dans l'extrémité opposée de l'ouverture. L'eau passée dans la première bouteille était claire, mais de plus en plus sale dans la deuxième et la troisième.

\section{Simona ReVigLio}

La rete "Essere Europa", di cui l'Istituto Matteotti di Rivoli (To) è capofila, ha coinvolto quest'anno nel progetto Erasmus Plus "L'Edd dans nos langues" gli studenti del Liceo linguistico Charles Darwin di Rivoli impegnati nell'alternanza scuola-lavoro.

L'obiettivo dell'attività programmata, che ha una durata complessiva di circa quattro mesi, è quello di accompagnare gli alunni della scuola primaria nella produzione di semplici video giochi didattici in lingua francese con l'aiuto dei compagni più grandi.

Il progetto ha previsto una formazione finalizzata all'acquisizione di competenze specifiche di progettazione e di creazione di giochi didattici multimediali che le insegnanti della scuola primaria e i giovani tirocinanti hanno svolto insieme con grande interesse. A questa formazione ha fatto seguito un altro percorso, specifico per i tirocinanti, di carattere pedagogico e didattico. L'approccio con i bambini richiede infatti la conoscenza dei processi di apprendimento dell'età evolutiva, dei tempi di attenzione relativi, delle tecniche di intervento più appropriate al fine di coinvolgere attivamente anche i più piccoli nello svolgimento dell'attività.

Conclusa la fase teorica, insegnanti e tirocinanti hanno iniziato gli incontri laboratoriali con i bambini per la realizzazione dei videogiochi.

I contenuti proposti per la costruzione dei giochi sono stati quelli che si erano stabiliti collegialmente nel seminario di novembre in Spagna: nello specifico, il nostro gruppo si è occupato della classificazione degli alimenti, della piramide alimentare e dell'importanza di un'alimentazione equilibrata per la crescita e lo sviluppo in età infantile.

Già dai primi incontri si è potuta apprezzare la validità dell'attività: i bambini, accompagnati dai loro "pari" si sono dimostrati più motivati e disposti ad imparare; gli alunni del liceo, diventati al tempo stesso tutor e mentori, hanno rivelato autonomia e capacità di iniziativa all'interno di un contesto per loro completamente nuovo. 


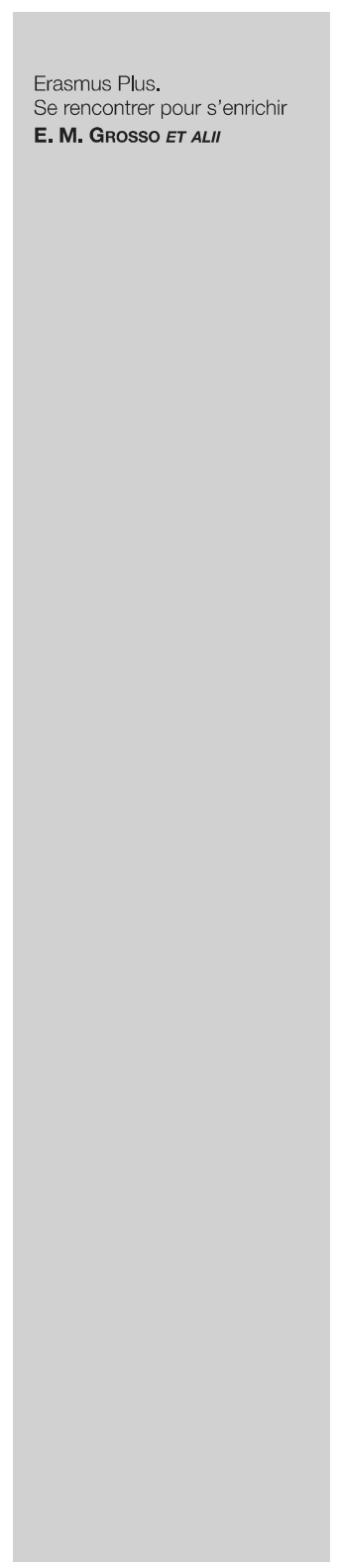

La valutazione e l'autovalutazione procedono in itinere: ad ogni attività in laboratorio segue un incontro di riflessione tra i ragazzi e il tutor di progetto e questo permette di rendere sempre più efficace l'esperienza per tutte le parti interessate.

I giochi verranno poi proposti a tutti i bambini delle nostre scuole che potranno così essere coinvolti in un'esperienza istruttiva $e$ divertente, che permetterà loro anche un approccio alla conoscenza della lingua francese. 